\title{
New Oscillatory Behavior of Third-Order Nonlinear Delay Dynamic Equations on Time Scales
}

\author{
Li Gao, Quanxin Zhang, and Shouhua Liu \\ Department of Mathematics, Binzhou University, Shandong 256603, China \\ Correspondence should be addressed to Li Gao; gaolibzxy@163.com
}

Received 18 December 2013; Accepted 22 January 2014; Published 11 March 2014

Academic Editor: Tongxing Li

Copyright (c) $2014 \mathrm{Li} \mathrm{Gao} \mathrm{et} \mathrm{al.} \mathrm{This} \mathrm{is} \mathrm{an} \mathrm{open} \mathrm{access} \mathrm{article} \mathrm{distributed} \mathrm{under} \mathrm{the} \mathrm{Creative} \mathrm{Commons} \mathrm{Attribution} \mathrm{License,} \mathrm{which}$ permits unrestricted use, distribution, and reproduction in any medium, provided the original work is properly cited.

A class of third-order nonlinear delay dynamic equations on time scales is studied. By using the generalized Riccati transformation and the inequality technique, four new sufficient conditions which ensure that every solution is oscillatory or converges to zero are established. The results obtained essentially improve earlier ones. Some examples are considered to illustrate the main results.

\section{Introduction}

In recent years, there has been much research activity concerning the oscillation and nonoscillation of solutions of various equations on time scales, and we refer the reader to the studies by Bohner and Saker [1] and Erbe et al. [2,3]. And there are some results dealing with oscillatory behavior of second-order delay dynamic equations on time scales [4-10]. However, there are few results dealing with the oscillation of the solutions of third-order delay dynamic equations on time scales, we refer the reader to the papers [11-14].

In this paper, we consider new oscillatory behavior of all solutions of the third-order nonlinear delay dynamic equation

$$
\begin{array}{r}
\left(r_{2}(t)\left[\left(r_{1}(t) x^{\Delta}(t)\right)^{\Delta}\right]^{\alpha}\right)^{\Delta}+q(t) f(x[\tau(t)])=0, \\
t \in \mathbb{T}, \quad t \geq t_{0},
\end{array}
$$

where $\alpha \geq 1$ is the ratio of two positive odd integers.

Throughout this paper, we will assume the following hypotheses.

$\left(H_{1}\right)$ T is a time scale (i.e., a nonempty closed subset of the real numbers $\mathbb{R}$ ) which is unbounded above, and $t_{0} \epsilon$ $\mathbb{T}$ with $t_{0}>0$, we define the time scale interval of the form $\left[t_{0}, \infty\right)_{\mathbb{T}}$ by $\left[t_{0}, \infty\right)_{\mathbb{T}}=\left[t_{0}, \infty\right) \cap \mathbb{T}$.
$\left(\mathrm{H}_{2}\right) r_{1}(t), r_{2}(t), q(t)$ are positive and real-valued rdcontinuous functions defined on $\mathbb{T}$, and $r_{1}(t), r_{2}(t)$ satisfy

$$
\int_{t_{0}}^{\infty} \frac{1}{r_{1}(s)} \Delta s=\infty, \quad \int_{t_{0}}^{\infty}\left(\frac{1}{r_{2}(s)}\right)^{1 / \alpha} \Delta s=\infty
$$

$\left(\mathrm{H}_{3}\right) \tau: \mathbb{T} \rightarrow \mathbb{T}$ is a strictly increasing and differentiable function, such that

$$
\tau(t) \leq t, \quad \lim _{t \rightarrow \infty} \tau(t)=\infty, \quad \tau(\mathbb{T})=\mathbb{T} .
$$

$\left(\mathrm{H}_{4}\right) f: \mathbb{R} \rightarrow \mathbb{R}$ is a continuous function and there exists some positive constant $L$ such that $f(x) / x^{\alpha} \geq L$ for all $x \neq 0$.

By a solution of (1), we mean a nontrivial function $x(t)$ satisfying (1) which has the properties $x(t) \in$ $C_{r d}^{1}\left(\left[T_{x}, \infty\right)_{\mathbb{T}}, \mathbb{R}\right)$ for $T_{x} \geq t_{0}$, and $r_{2}(t)\left[\left(r_{1}(t) x^{\Delta}(t)\right)^{\Delta}\right]^{\alpha} \in$ $C_{r d}^{1}\left(\left[T_{x}, \infty\right)_{\mathbb{T}}, \mathbb{R}\right)$. Our attention is restricted to those solutions of (1) which satisfy $\sup \{|x(t)|: t \geq T\}>0$ for all $T \geq T_{x}$, where $C_{r d}$ is the space of $r d$-continuous functions. A solution $x(t)$ of $(1)$ is said to be oscillatory on $\left[T_{x}, \infty\right)_{\mathbb{W}}$ if it is neither eventually positive nor eventually negative. Otherwise it is called nonoscillatory. The equation itself is called oscillatory if all its solutions are oscillatory. 
If $\alpha=1, \tau(t)=t$, then (1) simplifies to the third-order nonlinear dynamic equation

$$
\begin{array}{r}
\left(r_{2}(t)\left[\left(r_{1}(t) x^{\Delta}(t)\right)^{\Delta}\right]\right)^{\Delta}+q(t) f(x(t))=0, \\
t \in \mathbb{T}, \quad t \geq t_{0} .
\end{array}
$$

If, furthermore, $r_{1}(t)=r_{2}(t)=1, f(x)=x, \tau(t)=t$, then (1) reduces to the third-order linear dynamic equation

$$
x^{\Delta \Delta \Delta}(t)+q(t) x(t)=0, \quad t \in \mathbb{T}, t \geq t_{0} .
$$

If, in addition, $\alpha=1$, then (1) reduces to the nonlinear delay dynamic equation

$$
\begin{array}{r}
\left(r_{2}(t)\left[\left(r_{1}(t) x^{\Delta}(t)\right)^{\Delta}\right]\right)^{\Delta}+q(t) f(x[\tau(t)])=0, \\
t \in \mathbb{T}, \quad t \geq t_{0} .
\end{array}
$$

In 2005, Erbe et al. [11] considered the general thirdorder nonlinear dynamic equation (4). By employing the generalized Riccati transformation techniques, they established some sufficient conditions which ensure that every solution of (4) is oscillatory or converges to zero. In 2007, Erbe et al. [12] studied the third-order linear dynamic equation (5), and they obtained Hille and Nehari type oscillation criteria for (5). In 2011, Han et al. [13] extended and improved the results of [12], meanwhile obtaining some oscillatory criteria for (6). In 2014, Gao et al. [14] extended some results of $[12,13]$ to $(1)$. On this basis, we continue to discuss the oscillation of solutions of (1). By using the generalized Riccati transformation and the inequality technique, we obtain some new sufficient conditions which guarantee that every solution of (1) is oscillatory or converges to zero. Our results will improve some results that have been established in [11-14].

Throughout this paper, we will make use of the following product and quotient rules:

$$
\begin{gathered}
(f g)^{\Delta}(t)=f^{\Delta}(t) g(t)+f(\sigma(t)) g^{\Delta}(t) \\
=f(t) g^{\Delta}(t)+f^{\Delta}(t) g(\sigma(t)), \\
\left(\frac{f}{g}\right)^{\Delta}(t)=\frac{f^{\Delta}(t) g(t)-f(t) g^{\Delta}(t)}{g(t) g(\sigma(t))} \text { if } g g^{\sigma} \neq 0 .
\end{gathered}
$$

For $b, c \in \mathbb{T}$ and a differentiable function $f$, the Cauchy integral of $f^{\Delta}$ is defined by

$$
\int_{b}^{c} f^{\Delta}(t) \Delta t=f(c)-f(b) .
$$

The integration by parts formula reads

$$
\begin{aligned}
\int_{b}^{c} f^{\Delta}(t) g(t) \Delta t= & f(c) g(c)-f(b) g(b) \\
& -\int_{b}^{c} f^{\sigma}(t) g^{\Delta}(t) \Delta t
\end{aligned}
$$

and improper integrals are defined in the usual way by

$$
\int_{b}^{\infty} f(s) \Delta s=\lim _{t \rightarrow \infty} \int_{b}^{t} f(s) \Delta s .
$$

For more details, see $[15,16]$.

\section{Several Lemmas}

In this section we present several lemmas that will be needed in the proofs of our results in Section 3.

Lemma 1. Assume that $x(t)$ is an eventually positive solution of (1), then there exists $T \in\left[t_{0}, \infty\right)_{\mathbb{V}}$ such that either

$$
\begin{gathered}
\text { (I) } x(t)>0, \quad x^{\Delta}(t)>0, \quad\left(r_{1}(t) x^{\Delta}(t)\right)^{\Delta}>0, \\
\left(r_{2}(t)\left[\left(r_{1}(t) x^{\Delta}(t)\right)^{\Delta}\right]^{\alpha}\right)^{\Delta}<0, \quad t \in[T, \infty)_{\mathbb{T}},
\end{gathered}
$$

or

$$
\begin{aligned}
\text { (II) } x(t)>0, \quad x^{\Delta}(t)<0, \quad\left(r_{1}(t) x^{\Delta}(t)\right)^{\Delta}>0, \\
\left(r_{2}(t)\left[\left(r_{1}(t) x^{\Delta}(t)\right)^{\Delta}\right]^{\alpha}\right)^{\Delta}<0, \quad t \in[T, \infty)_{\mathbb{T}} .
\end{aligned}
$$

Proof. Assume that $x(t)$ is an eventually positive solution of (1), then there exists $T \in\left[t_{0}, \infty\right)_{\mathbb{T}}$ such that $x(t)>0$ and $x(\tau(t))>0$ for all $t \in[T, \infty)_{\mathbb{T}}$. From $(1)$; we obtain

$$
\begin{aligned}
\left(r_{2}(t)\left[\left(r_{1}(t) x^{\Delta}(t)\right)^{\Delta}\right]^{\alpha}\right)^{\Delta} & =-q(t) f(x(\tau(t))) \\
& \leq-L q(t) x^{\alpha}[\tau(t)]<0 .
\end{aligned}
$$

Hence, $r_{2}(t)\left[\left(r_{1}(t) x^{\Delta}(t)\right)^{\Delta}\right]^{\alpha}$ is decreasing and therefore eventually of one sign, so $\left(r_{1}(t) x^{\Delta}(t)\right)^{\Delta}$ is either eventually positive or eventually negative. We assert that $\left(r_{1}(t) x^{\Delta}(t)\right)^{\Delta}>0$ for all $t \in[T, \infty)_{\mathbb{T}}$.

If there exists $t_{1} \in[T, \infty)_{\mathbb{T}}$ such that $\left(r_{1}\left(t_{1}\right) x^{\Delta}\left(t_{1}\right)\right)^{\Delta}<0$, we get

$$
\begin{array}{r}
r_{2}(t)\left[\left(r_{1}(t) x^{\Delta}(t)\right)^{\Delta}\right]^{\alpha} \leq r_{2}\left(t_{1}\right)\left[\left(r_{1}\left(t_{1}\right) x^{\Delta}\left(t_{1}\right)\right)^{\Delta}\right]^{\alpha}<0, \\
t \in\left[t_{1}, \infty\right)_{\mathbb{T}} .
\end{array}
$$

Let $M=-r_{2}\left(t_{1}\right)\left[\left(r_{1}\left(t_{1}\right) x^{\Delta}\left(t_{1}\right)\right)^{\Delta}\right]^{\alpha}>0$, then

$$
\left(r_{1}(t) x^{\Delta}(t)\right)^{\Delta} \leq-M^{1 / \alpha} \frac{1}{\left(r_{2}(t)\right)^{1 / \alpha}} .
$$

Integrating (16) from $t_{1}$ to $t\left(t \in\left[t_{1}, \infty\right)_{\mathbb{T}}\right)$ provides

$$
\begin{aligned}
r_{1}(t) x^{\Delta}(t) \leq & r_{1}\left(t_{1}\right) x^{\Delta}\left(t_{1}\right)-M^{1 / \alpha} \\
& \times \int_{t_{1}}^{t} \frac{1}{\left(r_{2}(s)\right)^{1 / \alpha}} \Delta s \longrightarrow-\infty, \quad t \longrightarrow+\infty .
\end{aligned}
$$

Then there exists $t_{2} \in\left[t_{1}, \infty\right)_{\mathbb{T}}$ such that $r_{1}(t) x^{\Delta}(t) \leq$ $r_{1}\left(t_{2}\right) x^{\Delta}\left(t_{2}\right)<0$. Similarly, $t \in\left[t_{2}, \infty\right)_{\mathbb{T}}$, we obtain

$$
x(t) \leq x\left(t_{2}\right)+r_{1}\left(t_{2}\right) x^{\Delta}\left(t_{2}\right) \int_{t_{2}}^{t} \frac{1}{r_{1}(s)} \Delta s \longrightarrow-\infty,
$$


which contradicts with $x(t)>0$. So $\left(r_{1}(t) x^{\Delta}(t)\right)^{\Delta}>0$; this implies that $x^{\Delta}(t)>0$ or $x^{\Delta}(t)<0$ for all $t \in[T, \infty)_{\mathbb{T}}$. This completes the proof.

Lemma 2 (see [17]). Assume that $\left(\mathrm{H}_{3}\right)$ and the following conditions hold:

(I) $u(t) \in C_{r d}^{2}(I, \mathbb{R})$, where $I=\left[t^{*},+\infty\right)_{\mathbb{T}}$ for some $t^{*}>$ 0 ;

(II) $u(t)>0, u^{\Delta}(t)>0, u^{\Delta \Delta}(t) \leq 0$ for $t \geq t^{*}$.

Then, for each $k \in(0,1)$, there exists a constant $t_{k} \in \mathbb{T}, t_{k} \geq$ $t^{*}$ such that

$$
u(\sigma(t)) \leq \frac{\sigma(t) u(\tau(t))}{k \tau(t)} \text { for } t \geq t_{k} .
$$

Lemma 3 (see [15]). If $x$ is differentiable, then

$$
\left(x^{\gamma}\right)^{\Delta}=\gamma x^{\Delta} \int_{0}^{1}\left[h x^{\sigma}+(1-h) x\right]^{\gamma-1} d h .
$$

Lemma 4 (see [12]). Assume that $u$ satisfies

$$
\begin{gathered}
u(t)>0, \quad u^{\Delta}(t)>0, \quad u^{\Delta \Delta}(t)>0, \\
u^{\Delta \Delta \Delta}(t) \leq 0, \quad t \in[T, \infty)_{\mathbb{T}} .
\end{gathered}
$$

Then

$$
\liminf _{t \rightarrow \infty} \frac{t u(t)}{h_{2}\left(t, t_{0}\right) u^{\Delta}(t)} \geq 1
$$

where the Taylor monomials $h_{n}(t, s)_{n=0}^{+\infty}$ are defined recursively by

$$
\begin{array}{r}
h_{0}(t, s)=1, \quad h_{n+1}(t, s)=\int_{s}^{t} h_{n}(\tau, s) \Delta \tau, \\
t, s \in \mathbb{T}, \quad n \geq 1 .
\end{array}
$$

Lemma 5 (see [18]). Assume that $X$ and $Y$ are nonnegative real numbers. Then

$$
\lambda X Y^{\lambda-1}-X^{\lambda} \leq(\lambda-1) Y^{\lambda}, \quad \forall \lambda>1,
$$

where the equality holds if and only if $X=Y$.

Lemma 6. Assume that $x(t)$ is an eventually positive solution of (1) which satisfies case (II) in Lemma 1, if either

$$
\int_{t_{0}}^{\infty} q(s) \Delta s=\infty
$$

or

$$
\begin{gathered}
\int_{t_{0}}^{\infty} q(s) \Delta s<\infty, \\
\int_{t_{0}}^{\infty} \frac{1}{r_{1}(t)} \int_{t}^{\infty}\left[\frac{1}{r_{2}(s)} \int_{s}^{\infty} q(u) \Delta u\right]^{1 / \alpha} \Delta s \Delta t=\infty .
\end{gathered}
$$

Then $\lim _{t \rightarrow \infty} x(t)=0$.
Proof. Assume that $x(t)$ is an eventually positive solution of (1) which satisfies case (II) in Lemma 1. Then $x(t)$ is decreasing and $\lim _{t \rightarrow \infty} x(t)=l \geq 0$. If $l>0$; it is easy to see that there exists $t_{1} \in\left[t_{0}, \infty\right)_{\mathbb{T}}$ such that $x[\tau(t)] \geq x(t) \geq l>0$ for all $t \in\left[t_{1}, \infty\right)_{\mathbb{V}}$. From (14),

$$
\left(r_{2}(t)\left[\left(r_{1}(t) x^{\Delta}(t)\right)^{\Delta}\right]^{\alpha}\right)^{\Delta} \leq-L q(t) x^{\alpha}[\tau(t)] \leq-L l^{\alpha} q(t) .
$$

If (25) holds, then integrating (27) from $t_{1}$ to $t(t \in$ $\left.\left[t_{1}, \infty\right)_{\mathbb{T}}\right)$, we get

$$
\begin{gathered}
r_{2}(t)\left[\left(r_{1}(t) x^{\Delta}(t)\right)^{\Delta}\right]^{\alpha} \leq r_{2}\left(t_{1}\right)\left[\left(r_{1}\left(t_{1}\right) x^{\Delta}\left(t_{1}\right)\right)^{\Delta}\right]^{\alpha}-L l^{\alpha} \\
\times \int_{t_{1}}^{t} q(s) \Delta s \longrightarrow-\infty \\
(t \longrightarrow+\infty) .
\end{gathered}
$$

This is contrary to $\left(r_{1}(t) x^{\Delta}(t)\right)^{\Delta}>0$.

If (26) holds, then integrating (1) from $t$ to $\infty$, we get

$$
\begin{aligned}
&-r_{2}(t) {\left[\left(r_{1}(t) x^{\Delta}(t)\right)^{\Delta}\right]^{\alpha} } \\
& \leq-L \int_{t}^{\infty} q(s) x^{\alpha}[\tau(s)] \Delta s \\
& \leq-L l^{\alpha} \int_{t}^{\infty} q(s) \Delta s, \quad t \in\left[t_{1}, \infty\right)_{\mathbb{T}}
\end{aligned}
$$

and hence,

$$
-\left(r_{1}(t) x^{\Delta}(t)\right)^{\Delta} \leq-l\left[\frac{1}{r_{2}(t)} \int_{t}^{\infty} L q(s) \Delta s\right]^{1 / \alpha} .
$$

Again, integrating this inequality from $t$ to $\infty$, we obtain

$$
r_{1}(t) x^{\Delta}(t) \leq-l L^{1 / \alpha} \int_{t}^{\infty}\left[\frac{1}{r_{2}(s)} \int_{s}^{\infty} q(u) \Delta u\right]^{1 / \alpha} \Delta s .
$$

Finally, integrating the last inequality from $T$ to $t$, we get

$$
\begin{aligned}
x(t)-x(T) \leq & -l L^{1 / \alpha} \int_{T}^{t} \frac{1}{r_{1}(s)} \\
& \times \int_{s}^{\infty}\left[\frac{1}{r_{2}(u)} \int_{u}^{\infty} q(v) \Delta v\right]^{1 / \alpha} \Delta u \Delta s .
\end{aligned}
$$

Hence by (26), we obtain $\lim _{t \rightarrow \infty} x(t)=-\infty$, which contradicts $x(t)>0$. Thus, we get $l=0$. This completes the proof.

Lemma 7 (see [19]). Let $a, b \in \mathbb{T}$. Then for positive $r d$ continuous functions $f, g:[a, b] \rightarrow \mathbb{R}$, one has

$$
\int_{a}^{b}|f(s) g(s)| \Delta s \leq\left(\int_{a}^{b}|f(s)|^{p} \Delta s\right)^{1 / p}\left(\int_{a}^{b}|g(s)|^{q} \Delta s\right)^{1 / q},
$$

where $p>1$ and $1 / p+1 / q=1$. 


\section{Main Results}

New we state and prove the main results of this paper.

Theorem 8. Assume that $\left(H_{1}\right)-\left(H_{4}\right),(26)$, and $r_{2}^{\Delta}(t) \geq 0$ hold. Furthermore, suppose that there exists a positive function $\delta \in$ $C_{r d}^{1}\left(\left[t_{0}, \infty\right)_{\mathbb{T}},(0, \infty)\right)$ with $\delta^{\Delta}(t) \geq 0$, and for all sufficiently large $T$, there exists $T_{0}>T$, such that

$$
\begin{aligned}
& \limsup _{t \rightarrow \infty} \int_{T_{0}}^{t}\left\{Q(s)-\frac{r_{2}(s) \delta(s)}{(\alpha+1)^{\alpha+1} k^{\alpha^{2}}}\left[\frac{\delta^{\Delta}(s)}{\delta(s)}\right]^{\alpha+1}\left[\frac{\sigma(s)}{\tau(s)}\right]^{\alpha^{2}}\right\} \Delta s \\
& \quad=\infty,
\end{aligned}
$$

where $Q(t)=\operatorname{Lq}(t) \delta(\sigma(t))\left(k h_{2}\left(\tau(t), t_{0}\right) /\left(2 r_{1}(\tau(t)) \sigma(t)\right)\right)^{\alpha}$. Then every solution $x(t)$ of (1) is either oscillatory or converges to zero.

Proof. Assume that (1) has a nonoscillatory solution $x(t)$ on $\left[t_{0}, \infty\right)_{\mathbb{T}}$. Without loss of generality, we may assume that there exists sufficiently large $T \geq t_{0}$, such that $x(t)>0$ and $x(\tau(t))>0$ for all $t \in[T, \infty)_{\mathbb{T}}$. In the case when $x(t)$ is eventually negative, the proof is similar. By Lemma 1 , we see that $x(t)$ satisfies either case (I) or case (II).

If case (I) holds, then $x^{\Delta}(t)>0, t \in[T, \infty)_{\mathbb{T}}$. Define the function $W(t)$ by

$$
W(t)=\delta(t) r_{2}(t)\left(\frac{\left(r_{1}(t) x^{\Delta}(t)\right)^{\Delta}}{r_{1}(t) x^{\Delta}(t)}\right)^{\alpha}, \quad t \in[T, \infty)_{\mathbb{T}} .
$$

Then $W(t)>0$. By the product rule (7) and the quotient rule (8), we have

$$
\begin{aligned}
W^{\Delta}(t)= & \frac{\delta^{\Delta}(t)}{\delta(t)} W(t)-\delta(\sigma(t)) \frac{q(t) f(x(\tau(t)))}{\left[r_{1}(\sigma(t)) x^{\Delta}(\sigma(t))\right]^{\alpha}} \\
& -\delta(\sigma(t)) r_{2}(t)\left[\frac{\left(r_{1}(t) x^{\Delta}(t)\right)^{\Delta}}{r_{1}(t) x^{\Delta}(t)}\right]^{\alpha} \\
& \times \frac{\left[\left(r_{1}(t) x^{\Delta}(t)\right)^{\alpha}\right]^{\Delta}}{\left[r_{1}(\sigma(t)) x^{\Delta}(\sigma(t))\right]^{\alpha}} .
\end{aligned}
$$

Let $u(t)=r_{1}(t) x^{\Delta}(t)$, from case (I) in Lemma 1, we get $u(t)>$ $0, u^{\Delta}(t)>0$. In view of that

$$
\begin{aligned}
{\left[r_{2}(t)\left(r_{1}(t) x^{\Delta}(t)\right)^{\Delta}\right]^{\Delta} } & =\left[r_{2}(t) u^{\Delta}(t)\right]^{\Delta} \\
& =r_{2}^{\Delta}(t) u^{\Delta}(\sigma(t))+r_{2}(t) u^{\Delta \Delta}(t) \\
& <0
\end{aligned}
$$

and $r_{2}^{\Delta}(t) \geq 0$, it is not difficult to see that $u^{\Delta \Delta}(t)<0$. Thus, by Lemma 2 , for every $k \in(0,1)$, there exists $t_{1} \in[T, \infty)_{\mathbb{T}}$ with $t_{1} \geq \max \left\{t_{k}, T\right\}$, such that $u(\sigma(t)) \leq \sigma(t) u(\tau(t)) / k \tau(t) \leq$ $\sigma(t) u(t) / k \tau(t)$ for all $t \in\left[t_{1}, \infty\right)_{\mathbb{T}}$; this implies that

$$
\begin{aligned}
r_{1}(\sigma(t)) x^{\Delta}(\sigma(t)) & \leq \frac{\sigma(t) r_{1}(\tau(t)) x^{\Delta}(\tau(t))}{k \tau(t)} \\
& \leq \frac{\sigma(t) r_{1}(t) x^{\Delta}(t)}{k \tau(t)} .
\end{aligned}
$$

By Lemma 3, we get $\left[(u(t))^{\alpha}\right]^{\Delta} \geq \alpha u^{\Delta}(t) \int_{0}^{1}[h u+(1-$ h) $u]^{\alpha-1} d h=\alpha(u(t))^{\alpha-1} u^{\Delta}(t)$, that is,

$$
\left[\left(r_{1}(t) x^{\Delta}(t)\right)^{\alpha}\right]^{\Delta} \geq \alpha\left(r_{1}(t) x^{\Delta}(t)\right)^{\alpha-1}\left(r_{1}(t) x^{\Delta}(t)\right)^{\Delta}
$$

Using (38) and (39),

$$
\begin{aligned}
W^{\Delta}(t) \leq & \frac{\delta^{\Delta}(t)}{\delta(t)} W(t)-\frac{L q(t) x^{\alpha}(\tau(t)) \delta(\sigma(t))}{\left[r_{1}(\sigma(t)) x^{\Delta}(\sigma(t))\right]^{\alpha}} \\
& -\frac{\alpha \delta(\sigma(t)) r_{2}(t)\left[\left(r_{1}(t) x^{\Delta}(t)\right)^{\Delta}\right]^{\alpha+1}}{\left(r_{1}(t) x^{\Delta}(t)\right)\left(r_{1}(\sigma(t)) x^{\Delta}(\sigma(t))\right)^{\alpha}} \\
\leq & \frac{\delta^{\Delta}(t)}{\delta(t)} W(t)-\frac{L q(t) x^{\alpha}(\tau(t)) \delta(\sigma(t))}{\left[r_{1}(\sigma(t)) x^{\Delta}(\sigma(t))\right]^{\alpha}} \\
& -\frac{\alpha k^{\alpha} \tau^{\alpha}(t) r_{2}(t) \delta(\sigma(t))\left[\frac{\left(r_{1}(t) x^{\Delta}(t)\right)^{\Delta}}{\sigma_{1}(t) x^{\Delta}(t)}\right]^{\alpha+1}}{\leq} \\
& \frac{\delta^{\Delta}(t)}{\delta(t)} W(t)-\frac{L q(t) x^{\alpha}(\tau(t)) \delta(\sigma(t))}{\left[r_{1}(\sigma(t)) x^{\Delta}(\sigma(t))\right]^{\alpha}} \\
& -\frac{\alpha k^{\alpha} \tau^{\alpha}(t) r_{2}(t) \delta(\sigma(t)) W^{1+1 / \alpha}(t)}{\sigma^{\alpha}(t)\left(\delta(t) r_{2}(t)\right)^{1+1 / \alpha}} \\
\leq & \frac{\delta^{\Delta}(t)}{\delta(t)} W(t)-\frac{L q(t) x^{\alpha}(\tau(t)) \delta(\sigma(t))}{\left[r_{1}(\sigma(t)) x^{\Delta}(\sigma(t))\right]^{\alpha}} \\
\sigma^{\alpha}(t)\left(\delta(t) r_{2}(t)\right)^{1 / \alpha} & k^{\alpha}(t) W^{1+1 / \alpha}(t) \\
&
\end{aligned}
$$

Let $v(t)=\int_{T}^{t} r_{1}(s) x^{\Delta}(s) \Delta s$ for all $t \in(T, \infty)_{\mathbb{T}}$; it is easy to see that $v(t)>0, v^{\Delta}(t)>0, v^{\Delta \Delta}(t)>0, v^{\Delta \Delta \Delta}(t) \leq 0$. Thus, by Lemma 4 , there exists $t_{1 / 2} \in[T, \infty)_{\mathbb{T}}$ such that $t v(t) /\left(h_{2}\left(t, t_{0}\right) v^{\Delta}(t)\right) \geq 1 / 2$ for all $t \in\left[t_{1 / 2}, \infty\right)_{\mathbb{T}}$. Then, we get

$$
\frac{\int_{T}^{t} r_{1}(s) x^{\Delta}(s) \Delta s}{r_{1}(t) x^{\Delta}(t)} \geq \frac{h_{2}\left(t, t_{0}\right)}{2 t}, \quad t \in\left[t_{1 / 2}, \infty\right)_{\mathbb{T}} .
$$


From $\int_{T}^{t} r_{1}(s) x^{\Delta}(s) \Delta s=r_{1}(t) x(t)-r_{1}(T) x(T)-$ $\int_{T}^{t} r_{1}^{\Delta}(s) x^{\Delta}(\sigma(s)) \Delta s$, we obtain $r_{1}(t) x(t) \geq \int_{T}^{t} r_{1}(s) x^{\Delta}(s) \Delta s$. By (41), we get

$$
\begin{array}{r}
\frac{x(t)}{x^{\Delta}(t)}=\frac{r_{1}(t) x(t)}{r_{1}(t) x^{\Delta}(t)} \geq \frac{\int_{T}^{t} r_{1}(s) x^{\Delta}(s) \Delta s}{r_{1}(t) x^{\Delta}(t)} \geq \frac{h_{2}\left(t, t_{0}\right)}{2 t}, \\
t \in\left[t_{1 / 2}, \infty\right)_{\mathbb{T}} .
\end{array}
$$

Therefore, from (38) and (42), there exists $t_{2} \in\left[t_{0}, \infty\right)_{\mathbb{T}}$ with $t_{2} \geq \max \left\{t_{1}, t_{1 / 2}\right\}$ such that

$$
\frac{x(\tau(t))}{r_{1}(\sigma(t)) x^{\Delta}(\sigma(t))} \geq \frac{k h_{2}\left(\tau(t), t_{0}\right)}{2 r_{1}(\tau(t)) \sigma(t)}, \quad t \in\left[t_{2}, \infty\right)_{\mathbb{T}} .
$$

Using (43), we get

$$
\begin{aligned}
W^{\Delta}(t) \leq & -\frac{L k^{\alpha} q(t) \delta(\sigma(t)) h_{2}^{\alpha}\left(\tau(t), t_{0}\right)}{2^{\alpha}\left[r_{1}(\tau(t)) \sigma(t)\right]^{\alpha}}+\frac{\delta^{\Delta}(t)}{\delta(t)} W(t) \\
& -\frac{\alpha k^{\alpha} \tau^{\alpha}(t)}{\sigma^{\alpha}(t)\left(\delta(t) r_{2}(t)\right)^{1 / \alpha}} W^{1+1 / \alpha}(t), \quad t \in\left[t_{2}, \infty\right)_{\mathbb{T}}
\end{aligned}
$$

that is,

$$
\begin{aligned}
Q(t) \leq & -W^{\Delta}(t)+\frac{\delta^{\Delta}(t)}{\delta(t)} W(t) \\
& -\frac{\alpha k^{\alpha} \tau^{\alpha}(t)}{\sigma^{\alpha}(t)\left(\delta(t) r_{2}(t)\right)^{1 / \alpha}} W^{1+1 / \alpha}(t) .
\end{aligned}
$$

Now, set

$$
\begin{gathered}
X^{\lambda}=\frac{\alpha k^{\alpha} \tau^{\alpha}(t)}{\sigma^{\alpha}(t)\left(\delta(t) r_{2}(t)\right)^{1 / \alpha}} W^{\lambda}(t), \\
Y^{\lambda-1}=\frac{\delta^{\Delta}(t)\left[\sigma^{\alpha}(t)\left(\delta(t) r_{2}(t)\right)^{1 / \alpha}\right]^{1 / \lambda}}{\lambda \delta(t)\left[\alpha k^{\alpha} \tau^{\alpha}(t)\right]^{1 / \lambda}},
\end{gathered}
$$

where $\lambda=(\alpha+1) / \alpha>1, X \geq 0$ and $Y \geq 0$. Using the equality (24), we obtain

$$
\begin{gathered}
\frac{\delta^{\Delta}(t)}{\delta(t)} W(t)-\frac{\alpha k^{\alpha} \tau^{\alpha}(t)}{\sigma^{\alpha}(t)\left(\delta(t) r_{2}(t)\right)^{1 / \alpha}} W^{1+1 / \alpha}(t) \\
\quad \leq \frac{r_{2}(t) \delta(t)}{(\alpha+1)^{\alpha+1} k^{\alpha^{2}}}\left[\frac{\delta^{\Delta}(t)}{\delta(t)}\right]^{\alpha+1}\left[\frac{\sigma(t)}{\tau(t)}\right]^{\alpha^{2}} .
\end{gathered}
$$

From (47), we obtain

$$
Q(t) \leq-W^{\Delta}(t)+\frac{r_{2}(t) \delta(t)}{(\alpha+1)^{\alpha+1} k^{\alpha^{2}}}\left[\frac{\delta^{\Delta}(t)}{\delta(t)}\right]^{\alpha+1}\left[\frac{\sigma(t)}{\tau(t)}\right]^{\alpha^{2}}
$$

Integrating (48) from $T_{0}$ to $t$, we get

$$
\begin{aligned}
\int_{T_{0}}^{t} Q(s) \Delta s \leq & W\left(T_{0}\right)-W(t) \\
& +\int_{T_{0}}^{t} \frac{r_{2}(s) \delta(s)}{(\alpha+1)^{\alpha+1} k^{\alpha^{2}}}\left[\frac{\delta^{\Delta}(s)}{\delta(s)}\right]^{\alpha+1}\left[\frac{\sigma(s)}{\tau(s)}\right]^{\alpha^{2}} \Delta s,
\end{aligned}
$$

consequently,

$$
\begin{gathered}
\int_{T_{0}}^{t}\left\{Q(s)-\frac{r_{2}(s) \delta(s)}{(\alpha+1)^{\alpha+1} k^{\alpha^{2}}}\left[\frac{\delta^{\Delta}(s)}{\delta(s)}\right]^{\alpha+1}\left[\frac{\sigma(s)}{\tau(s)}\right]^{\alpha^{2}}\right\} \Delta s \\
\leq W\left(T_{0}\right)-W(t) \leq W\left(T_{0}\right) .
\end{gathered}
$$

This is contrary to (34).

If case (II) holds, from (26), we get $\lim _{t \rightarrow \infty} x(t)=0$. This completes the proof.

Theorem 9. Assume that $\left(H_{1}\right)-\left(H_{4}\right),(26)$, and $r_{2}^{\Delta}(t) \geq 0$ hold. Furthermore, suppose that there exist functions $H, h \in$ $C_{r d}(\mathbb{D}, \mathbb{R})$, where $\mathbb{D} \equiv\{(t, s): t \geq s \geq T\}$ such that

$$
\begin{gathered}
H(t, t)=0, \quad t \geq T \\
H(t, s)>0, \quad t>s \geq T,
\end{gathered}
$$

and $H$ has a nompositive continuous $\Delta$-partial derivative $H^{\Delta_{s}}(t, s)$ on $\mathbb{D}$ with respect to the second variable and satisfies, for all sufficiently large $T \in\left[t_{0}, \infty\right)_{\mathbb{T}}$, that there exists $T_{0}>T$, such that

$$
\begin{aligned}
H^{\Delta_{s}} & (\sigma(t), s)+\frac{\delta^{\Delta}(s)}{\delta(s)} H(\sigma(t), s) \\
& =-\frac{h(t, s)}{\delta(s)} H^{\alpha /(\alpha+1)}(\sigma(t), s) \quad \text { for }(t, s) \in \mathbb{D},
\end{aligned}
$$

$$
\begin{aligned}
& \limsup _{t \rightarrow \infty} \frac{1}{H\left(\sigma(t), T_{0}\right)} \\
& \quad \times \int_{T_{0}}^{\sigma(t)}\left[H(\sigma(t), s) Q(s)-\frac{h_{-}^{\alpha+1}(t, s) r_{2}(s) \sigma^{\alpha^{2}}(s)}{(\alpha+1)^{\alpha+1} k^{\alpha^{2}} \tau^{\alpha^{2}}(s) \delta^{\alpha}(s)}\right] \Delta s
\end{aligned}
$$

$=\infty$,

where $\delta(t)$ and $Q(t)$ are defined in Theorem 8. $h_{-}(t, s)=$ $\max \{0,-h(t, s)\}, h_{+}(t, s)=\max \{0, h(t, s)\}$. Then every solution $x(t)$ of (1) is either oscillatory or converges to zero.

Proof. Assume that (1) has a nonoscillatory solution $x(t)$ on $\left[t_{0}, \infty\right)_{\mathbb{T}}$. Without loss of generality, we may assume that there exists sufficiently large $T \geq t_{0}$, such that $x(t)>0$ and $x[\tau(t)]>0$ for all $t \in[T, \infty)_{\mathbb{T}}$. In the case when $x(t)$ is eventually negative, the proof is similar. By Lemma 1 , we see that $x(t)$ satisfies either case (I) or case (II). If case (I) holds, we proceed as in the proof of Theorem 8 and get (45). In 
(45), replace $t$ by $s$ and multiply both sides by $H(\sigma(t), s)$ and integrate with respect to $s$ from $T_{0}$ to $\sigma(t), t \geq T_{0}$; we get

$$
\begin{aligned}
\int_{T_{0}}^{\sigma(t)} & H(\sigma(t), s) Q(s) \Delta s \\
\leq & -\int_{T_{0}}^{\sigma(t)} H(\sigma(t), s) W^{\Delta}(s) \Delta s \\
& +\int_{T_{0}}^{\sigma(t)} H(\sigma(t), s) \frac{\delta^{\Delta}(s)}{\delta(s)} W(s) \Delta s \\
& -\int_{T_{0}}^{\sigma(t)} H(\sigma(t), s) \times \frac{\alpha k^{\alpha} \tau^{\alpha}(s)}{\sigma^{\alpha}(s)\left(\delta(s) r_{2}(s)\right)^{1 / \alpha}} \\
& \times W^{1+1 / \alpha}(s) \Delta s .
\end{aligned}
$$

Integrating by parts using (51) and (52), we obtain

$$
\begin{aligned}
\int_{T_{0}}^{\sigma(t)} & H(\sigma(t), s) Q(s) \Delta s \\
\leq & H\left(\sigma(t), T_{0}\right) W\left(T_{0}\right)+\int_{T_{0}}^{\sigma(t)} H^{\Delta_{s}}(\sigma(t), s) W(s) \Delta s \\
& +\int_{T_{0}}^{\sigma(t)} \frac{H(\sigma(t), s) \delta^{\Delta}(s)}{\delta(s)} W(s) \Delta s \\
& -\int_{T_{0}}^{\sigma(t)} \frac{\alpha k^{\alpha} H(\sigma(t), s) \tau^{\alpha}(s)}{\sigma^{\alpha}(s)\left(\delta(s) r_{2}(s)\right)^{1 / \alpha}} W^{1+1 / \alpha}(s) \Delta s \\
\leq & H\left(\sigma(t), T_{0}\right) W\left(T_{0}\right) \\
& +\int_{T_{0}}^{\sigma(t)}\left[-\frac{h(t, s) H^{\alpha /(1+\alpha)}(\sigma(t), s)}{\delta(s)} W(s)\right. \\
& \left.-\frac{\alpha k^{\alpha} H(\sigma(t), s) \tau^{\alpha}(s)}{\sigma^{\alpha}(s)\left(\delta(s) r_{2}(s)\right)^{1 / \alpha}} W^{1+1 / \alpha}(s)\right] \Delta s,
\end{aligned}
$$

where $\lambda=(\alpha+1) / \alpha>1, X \geq 0$ and $Y \geq 0$. Using inequality (24), we obtain

$$
\begin{gathered}
\frac{h_{-}(t, s) H^{1 / \lambda}(\sigma(t), s)}{\delta(s)} W(s)-\frac{\alpha k^{\alpha} H(\sigma(t), s) \tau^{\alpha}(s)}{\sigma^{\alpha}(s)\left(\delta(s) r_{2}(s)\right)^{1 / \alpha}} W^{\lambda}(s) \\
\leq \frac{h_{-}^{\alpha+1}(t, s) r_{2}(s) \sigma^{\alpha^{2}}(s)}{(\alpha+1)^{\alpha+1} k^{\alpha^{2}} \tau^{\alpha^{2}}(s) \delta^{\alpha}(s)} .
\end{gathered}
$$

Combining (56) and (58), we get

$$
\begin{aligned}
& \frac{1}{H\left(\sigma(t), T_{0}\right)} \\
& \quad \times \int_{T_{0}}^{\sigma(t)}\left[H(\sigma(t), s) Q(s)-\frac{h_{-}^{\alpha+1}(t, s) r_{2}(s) \sigma^{\alpha^{2}}(s)}{(\alpha+1)^{\alpha+1} k^{\alpha^{2}} \tau^{\alpha^{2}}(s) \delta^{\alpha}(s)}\right] \Delta s \\
& \leq W\left(T_{0}\right),
\end{aligned}
$$

which contradicts (53).

If case (II) holds, from (26), we get $\lim _{t \rightarrow \infty} x(t)=0$. This completes the proof.

If (53) is not held, then we get the following result.

Theorem 10. Assume that $\left(H_{1}\right)-\left(H_{4}\right),(26)$, and $r_{2}^{\Delta}(t) \geq 0$ hold. Furthermore, suppose that there exist functions $H, h \in$ $C_{r d}(\mathbb{D}, \mathbb{R})$, where $\mathbb{D} \equiv\{(t, s): t \geq s \geq T\}$, such that (51) holds, $H$ has a nonpositive continuous $\Delta$-partial derivative $H^{\Delta_{s}}(t, s)$ on $\mathbb{D}$ with respect to the second variable and satisfies (52). Assume that

$$
0<\inf _{s \geq T_{0}}\left[\liminf _{t \rightarrow \infty} \frac{H(\sigma(t), s)}{H\left(\sigma(t), T_{0}\right)}\right] \leq \infty, \quad T_{0} \in\left[t_{0}, \infty\right)_{\mathbb{T}},
$$

$$
\limsup _{t \rightarrow \infty} \frac{1}{H\left(\sigma(t), T_{0}\right)} \int_{T_{0}}^{\sigma(t)} \frac{h_{-}^{\alpha+1}(t, s) r_{2}(s) \sigma^{\alpha^{2}}(s)}{\tau^{\alpha^{2}}(s) \delta^{\alpha}(s)} \Delta s<\infty
$$


and a real rd-continuous function $\Psi:\left[t_{0}, \infty\right)_{\mathbb{T}} \rightarrow \mathbb{R}$ such that

$$
\int_{T}^{\infty} \frac{\tau^{\alpha}(s)}{\sigma^{\alpha}(s)\left(\delta(s) r_{2}(s)\right)^{1 / \alpha}} \Psi_{+}^{1+1 / \alpha}(s) \Delta s=\infty,
$$

$$
\begin{aligned}
& \limsup _{t \rightarrow \infty} \frac{1}{H\left(\sigma(t), T_{0}\right)} \\
& \quad \times \int_{T_{0}}^{\sigma(t)}\left[H(\sigma(t), s) Q(s)-\frac{h_{-}^{\alpha+1}(t, s) r_{2}(s) \sigma^{\alpha^{2}}(s)}{(\alpha+1)^{\alpha+1} k^{\alpha^{2}} \tau^{\alpha^{2}}(s) \delta^{\alpha}(s)}\right] \Delta s \\
& \geq \Psi\left(T_{0}\right)
\end{aligned}
$$

for $T_{0} \in(T, \infty)_{\mathbb{T}}$, where $\delta(t)$ and $Q(t)$ are defined in Theorem $8, \Psi_{+}(t)=\max \{0, \Psi(t)\}$. Then every solution $x(t)$ of (1) is either oscillatory or converges to zero.

Proof. Assume that (1) has a nonoscillatory solution $x(t)$ on $\left[t_{0}, \infty\right)_{\mathbb{T}}$. Without loss of generality, we may assume that there exists sufficiently large $T \geq t_{0}$, such that $x(t)>0$ and $x[\tau(t)]>0$ for all $t \in[T, \infty)_{\mathbb{T}}$. In the case when $x(t)$ is eventually negative, the proof is similar. By Lemma 1 , we see that $x(t)$ satisfies either case (I) or case (II).

If case (I) holds, proceeding as in the proof of Theorem 9, we get that (56) and (58) hold. Then we conclude that

$$
\begin{aligned}
& \frac{1}{H\left(\sigma(t), T_{0}\right)} \\
& \quad \times \int_{T_{0}}^{\sigma(t)}\left[H(\sigma(t), s) Q(s)-\frac{h_{-}^{\alpha+1}(t, s) r_{2}(s) \sigma^{\alpha^{2}}(s)}{(\alpha+1)^{\alpha+1} k^{\alpha^{2}} \tau^{\alpha^{2}}(s) \delta^{\alpha}(s)}\right] \Delta s \\
& \leq W\left(T_{0}\right) .
\end{aligned}
$$

From (63), we obtain

$$
\begin{gathered}
\Psi\left(T_{0}\right) \leq W\left(T_{0}\right), \quad T_{0} \in(T, \infty)_{\mathbb{T}} \\
\limsup _{t \rightarrow \infty} \frac{1}{H\left(\sigma(t), T_{0}\right)} \int_{T_{0}}^{\sigma(t)} H(\sigma(t), s) Q(s) \Delta s \geq \Psi\left(T_{0}\right) .
\end{gathered}
$$

By (56), we get

$$
\begin{aligned}
& \frac{1}{H\left(\sigma(t), T_{0}\right)} \int_{T_{0}}^{\sigma(t)} H(\sigma(t), s) Q(s) \Delta s \\
& \leq W\left(T_{0}\right)+\frac{1}{H\left(\sigma(t), T_{0}\right)} \\
& \quad \times \int_{T_{0}}^{\sigma(t)} \frac{h_{-}(t, s) H^{\alpha /(1+\alpha)}(\sigma(t), s)}{\delta(s)} W(s) \Delta s \\
& \quad-\frac{1}{H\left(\sigma(t), T_{0}\right)} \\
& \quad \times \int_{T_{0}}^{\sigma(t)} \frac{\alpha k^{\alpha} H(\sigma(t), s) \tau^{\alpha}(s)}{\sigma^{\alpha}(s)\left(\delta(s) r_{2}(s)\right)^{1 / \alpha}} W^{1+1 / \alpha}(s) \Delta s .
\end{aligned}
$$

We denote

$$
\begin{aligned}
A(t)= & \frac{1}{H\left(\sigma(t), T_{0}\right)} \\
& \times \int_{T_{0}}^{\sigma(t)} \frac{h_{-}(t, s) H^{\alpha /(1+\alpha)}(\sigma(t), s)}{\delta(s)} W(s) \Delta s, \\
B(t)= & \frac{1}{H\left(\sigma(t), T_{0}\right)} \\
& \times \int_{T_{0}}^{\sigma(t)} \frac{\alpha k^{\alpha} H(\sigma(t), s) \tau^{\alpha}(s)}{\sigma^{\alpha}(s)\left(\delta(s) r_{2}(s)\right)^{1 / \alpha}} W^{1+1 / \alpha}(s) \Delta s,
\end{aligned}
$$

meanwhile noting that (63), we obtain

$$
\liminf _{t \rightarrow \infty}[B(t)-A(t)] \leq W\left(T_{0}\right)-\Psi\left(T_{0}\right)<\infty
$$

Now we assert that

$$
\int_{T}^{\infty} \frac{\tau^{\alpha}(s)}{\sigma^{\alpha}(s)\left(\delta(s) r_{2}(s)\right)^{1 / \alpha}} W^{1+1 / \alpha}(s) \Delta s<\infty
$$

holds. Suppose to the contrary that

$$
\int_{T}^{\infty} \frac{\tau^{\alpha}(s)}{\sigma^{\alpha}(s)\left(\delta(s) r_{2}(s)\right)^{1 / \alpha}} W^{1+1 / \alpha}(s) \Delta s=\infty
$$

by (60), there exists a constant $\varepsilon>0$ such that

$$
\inf _{s \geq T_{0}}\left[\liminf _{t \rightarrow \infty} \frac{H(\sigma(t), s)}{H\left(\sigma(t), T_{0}\right)}\right]>\varepsilon>0,
$$

from (71); there exists $T_{1} \in\left[T_{0}, \infty\right)_{\mathbb{T}}$ for arbitrary real number $M>0$ such that

$$
\begin{array}{r}
\int_{T_{1}}^{\sigma(t)} \frac{\tau^{\alpha}(s)}{\sigma^{\alpha}(s)\left(\delta(s) r_{2}(s)\right)^{1 / \alpha}} W^{1+1 / \alpha}(s) \Delta s \geq \frac{M}{\alpha k^{\alpha} \varepsilon}, \\
\text { for } t \in\left[T_{1}, \infty\right)_{\mathbb{T}} .
\end{array}
$$


By (10), we obtain

$$
\begin{aligned}
& B(t)=\frac{1}{H\left(\sigma(t), T_{0}\right)} \\
& \times \int_{T_{0}}^{\sigma(t)}\left\{\alpha k^{\alpha} H(\sigma(t), s)\right. \\
& \times\left(\int_{T_{0}}^{s} \frac{\tau^{\alpha}(u)}{\sigma^{\alpha}(u)\left(\delta(u) r_{2}(u)\right)^{1 / \alpha}}\right. \\
& \left.\left.\times W^{1+1 / \alpha}(u) \Delta u\right)^{\Delta_{s}}\right\} \Delta s \\
& =\frac{1}{H\left(\sigma(t), T_{0}\right)} \\
& \times \int_{T_{0}}^{\sigma(t)}\left\{-\alpha k^{\alpha} H^{\Delta_{s}}(\sigma(t), \sigma(s))\right. \\
& \times \int_{T_{0}}^{\sigma(s)} \frac{\tau^{\alpha}(u)}{\sigma^{\alpha}(u)\left(\delta(u) r_{2}(u)\right)^{1 / \alpha}} \\
& \left.\times W^{1+1 / \alpha}(u) \Delta u\right\} \Delta s \\
& \geq \frac{1}{H\left(\sigma(t), T_{0}\right)} \\
& \times \int_{T_{1}}^{\sigma(t)}\left\{-\alpha k^{\alpha} H^{\Delta_{s}}(\sigma(t), s)\right. \\
& \times \int_{T_{0}}^{\sigma(s)} \frac{\tau^{\alpha}(u)}{\sigma^{\alpha}(u)\left(\delta(u) r_{2}(u)\right)^{1 / \alpha}} \\
& \left.\times W^{1+1 / \alpha}(u) \Delta u\right\} \Delta s \\
& \geq \frac{1}{H\left(\sigma(t), T_{0}\right)} \int_{T_{1}}^{\sigma(t)}-\alpha k^{\alpha} H^{\Delta_{s}}(\sigma(t), s) \frac{M}{\alpha k^{\alpha} \varepsilon} \Delta s \\
& =\frac{M}{\varepsilon} \frac{H\left(\sigma(t), T_{1}\right)}{H\left(\sigma(t), T_{0}\right)} .
\end{aligned}
$$

From (72), there exists $T_{2} \in\left[T_{1}, \infty\right)_{\mathbb{T}}$, we get $H(\sigma(t)$, $\left.T_{1}\right) / H\left(\sigma(t), T_{0}\right) \geq \varepsilon$ for $t \in\left[T_{2}, \infty\right)_{\mathbb{T}}$, so that $B(t) \geq M$. Since $M$ is arbitrary, we obtain

$$
\lim _{t \rightarrow \infty} B(t)=\infty .
$$

Selecting a sequence $\left\{T_{n}\right\}_{n=1}^{\infty}: T_{n} \in\left[T_{0}, \infty\right)_{\mathbb{T}}$ with $\lim _{n \rightarrow \infty} T_{n}=\infty$ satisfying

$$
\lim _{n \rightarrow \infty}\left[B\left(T_{n}\right)-A\left(T_{n}\right)\right]=\liminf _{t \rightarrow \infty}[B(t)-A(t)]<\infty,
$$

then there exists a constant $M_{0}>0$ such that

$$
B\left(T_{n}\right)-A\left(T_{n}\right) \leq M_{0}
$$

for sufficiently large positive integer $n$. From (75), we can easily see

$$
\lim _{n \rightarrow \infty} B\left(T_{n}\right)=\infty
$$

(77) implies that

$$
\lim _{n \rightarrow \infty} A\left(T_{n}\right)=\infty
$$

From (77) and (78), we obtain

$$
\frac{A\left(T_{n}\right)}{B\left(T_{n}\right)}-1 \geq-\frac{M_{0}}{B\left(T_{n}\right)}>-\frac{M_{0}}{2 M_{0}}=-\frac{1}{2},
$$

that is,

$$
\frac{A\left(T_{n}\right)}{B\left(T_{n}\right)}>\frac{1}{2}
$$

for sufficiently large positive integer $n$, which together with (79) implies

$$
\lim _{n \rightarrow \infty} \frac{\left[A\left(T_{n}\right)\right]^{\alpha+1}}{\left[B\left(T_{n}\right)\right]^{\alpha}}=\lim _{n \rightarrow \infty}\left[\frac{A\left(T_{n}\right)}{B\left(T_{n}\right)}\right]^{\alpha} A\left(T_{n}\right)=\infty .
$$

On the other hand, by Lemma 7, we obtain

$$
\begin{aligned}
& A\left(T_{n}\right) \\
& =\frac{1}{H\left(\sigma\left(T_{n}\right), T_{0}\right)} \\
& \times \int_{T_{0}}^{\sigma\left(T_{n}\right)} \frac{h_{-}\left(T_{n}, s\right) H^{\alpha /(\alpha+1)}\left(\sigma\left(T_{n}\right), s\right)}{\delta(s)} W(s) \Delta s \\
& =\int_{T_{0}}^{\sigma\left(T_{n}\right)} \frac{h_{-}\left(T_{n}, s\right) H^{\alpha /(\alpha+1)}\left(\sigma\left(T_{n}\right), s\right)}{H\left(\sigma\left(T_{n}\right), T_{0}\right) \delta(s)} W(s) \Delta s \\
& =\int_{T_{0}}^{\sigma\left(T_{n}\right)}\left\{\left[\frac{\alpha k^{\alpha} H\left(\sigma\left(T_{n}\right), s\right) \tau^{\alpha}(s)}{H\left(\sigma\left(T_{n}\right), T_{0}\right)}\right]^{\alpha /(\alpha+1)}\right. \\
& \left.\times \frac{W(s)}{\sigma^{\alpha^{2} /(\alpha+1)}(s)\left(\delta(s) r_{2}(s)\right)^{1 /(\alpha+1)}}\right\} \\
& \times\left\{\left(h_{-}\left(T_{n}, s\right) H^{\alpha /(\alpha+1)}\left(\sigma\left(T_{n}\right), s\right)\right.\right. \\
& \left.\times \sigma^{\alpha^{2} /(\alpha+1)}(s) r_{2}^{1 /(\alpha+1)}(s)\right) \\
& \times\left(H\left(\sigma\left(T_{n}\right), T_{0}\right) \delta^{\alpha /(\alpha+1)}(s)\right)^{-1} \\
& \left.\times\left[\frac{\alpha k^{\alpha} H\left(\sigma\left(T_{n}\right), s\right) \tau^{\alpha}(s)}{H\left(\sigma\left(T_{n}\right), T_{0}\right)}\right]^{-\alpha /(\alpha+1)}\right\} \Delta s
\end{aligned}
$$




$$
\begin{aligned}
& \leq\left\{\int_{T_{0}}^{\sigma\left(T_{n}\right)} \frac{\alpha k^{\alpha} H\left(\sigma\left(T_{n}\right), s\right) \tau^{\alpha}(s)}{H\left(\sigma\left(T_{n}\right), T_{0}\right)}\right. \\
& \left.\quad \times\left[\frac{W(s)}{\sigma^{\alpha^{2} /(\alpha+1)}(s)\left(\delta(s) r_{2}(s)\right)^{1 /(\alpha+1)}}\right]^{(\alpha+1) / \alpha} \Delta s\right\}^{\alpha /(\alpha+1)} \\
& \times\left\{\int_{T_{0}}^{\sigma\left(T_{n}\right)} \frac{h_{-}^{\alpha+1}\left(T_{n}, s\right) H^{\alpha}\left(\sigma\left(T_{n}\right), s\right) \sigma^{\alpha^{2}}(s) r_{2}(s)}{H^{\alpha+1}\left(\sigma\left(T_{n}\right), T_{0}\right) \delta^{\alpha}(s)}\right. \\
& \left.\left.=\left[B\left(T_{n}\right)\right]^{\alpha /(\alpha+1)}\right]^{-\alpha}\right\}^{1 /(\alpha+1)} \\
& \times\left\{\frac{H^{\alpha}\left(\sigma\left(T_{n}\right), T_{0}\right)}{\alpha^{\alpha} k^{\alpha^{2}} H\left(\sigma\left(T_{n}\right), T_{0}\right)}\right. \\
& \left.\times \int_{T_{0}}^{\sigma\left(T_{n}\right)} \frac{h_{-}^{\alpha+1}\left(T_{n}, s\right) \sigma^{\alpha^{2}}(s) r_{2}(s)}{\delta^{\alpha}(s) \tau^{\alpha^{2}}(s)} \Delta s\right\}^{1 /(\alpha+1)}
\end{aligned}
$$

The above inequality shows that

$$
\begin{aligned}
\frac{\left[A\left(T_{n}\right)\right]^{\alpha+1}}{\left[B\left(T_{n}\right)\right]^{\alpha}} \leq & \frac{1}{\alpha^{\alpha} k^{\alpha^{2}} H\left(\sigma\left(T_{n}\right), T_{0}\right)} \\
& \times \int_{T_{0}}^{\sigma\left(T_{n}\right)} \frac{h_{-}^{\alpha+1}\left(T_{n}, s\right) \sigma^{\alpha^{2}}(s) r_{2}(s)}{\delta^{\alpha}(s) \tau^{\alpha^{2}}(s)} \Delta s .
\end{aligned}
$$

Hence, (82) implies

$$
\lim _{n \rightarrow \infty} \frac{1}{H\left(\sigma\left(T_{n}\right), T_{0}\right)} \int_{T_{0}}^{\sigma\left(T_{n}\right)} \frac{h_{-}^{\alpha+1}\left(T_{n}, s\right) r_{2}(s) \sigma^{\alpha^{2}}(s)}{\tau^{\alpha^{2}}(s) \delta^{\alpha}(s)} \Delta s=\infty .
$$

This contradicts (61). Therefore (70) holds. Noting $\Psi\left(T_{0}\right) \leq$ $W\left(T_{0}\right)$ for $T_{0} \in[T, \infty)_{\mathbb{T}}$, by using $(70)$, we obtain

$$
\begin{aligned}
\int_{T}^{\infty} & \frac{\tau^{\alpha}(s)}{\sigma^{\alpha}(s)\left(\delta(s) r_{2}(s)\right)^{1 / \alpha}} \Psi_{+}^{1+1 / \alpha}(s) \Delta s \\
& \leq \int_{T}^{\infty} \frac{\tau^{\alpha}(s)}{\sigma^{\alpha}(s)\left(\delta(s) r_{2}(s)\right)^{1 / \alpha}} W^{1+1 / \alpha}(s) \Delta s<\infty
\end{aligned}
$$

This contradicts (62). This completes the proof.

If case (II) holds, from (26), we get $\lim _{t \rightarrow \infty} x(t)=0$. This completes the proof.

Theorem 11. Assume that $\left(H_{1}\right)-\left(H_{4}\right),(26),(52),(60),(62)$, and $r_{2}^{\Delta}(t) \geq 0$ hold, where $H, h$, and $\delta$ are defined in
Theorem 10. Furthermore suppose that there is a real $\mathrm{rd}$ continuous function $\Psi:\left[t_{0}, \infty\right)_{\mathbb{V}} \rightarrow \mathbb{R}$ such that

$$
\begin{aligned}
& \liminf _{t \rightarrow \infty} \frac{1}{H\left(\sigma(t), T_{0}\right)} \int_{T_{0}}^{\sigma(t)} H(\sigma(t), s) Q(s) \Delta s<\infty, \quad(87) \\
& \liminf _{t \rightarrow \infty} \frac{1}{H\left(\sigma(t), T_{0}\right)} \\
& \quad \times \int_{T_{0}}^{\sigma(t)}\left[H(\sigma(t), s) Q(s)-\frac{h_{-}^{\alpha+1}(t, s) r_{2}(s) \sigma^{\alpha^{2}}(s)}{(\alpha+1)^{\alpha+1} k^{\alpha^{2}} \tau^{\alpha^{2}}(s) \delta^{\alpha}(s)}\right] \Delta s \\
& \geq \Psi\left(T_{0}\right)
\end{aligned}
$$

for $T_{0} \in(T, \infty)_{\mathbb{T}}$, where $Q(t)$ is defined in Theorem 8 , $\Psi_{+}(t)=\max \{0, \Psi(t)\}$. Then every solution $x(t)$ of $(1)$ is either oscillatory or converges to zero.

Proof. Assume that (1) has a nonoscillatory solution $x(t)$ on $\left[t_{0}, \infty\right)_{\mathbb{T}}$. Without loss of generality, we may assume that there exists sufficiently large $T \geq t_{0}$, such that $x(t)>0$ and $x[\tau(t)]>0$ for all $t \in[T, \infty)_{\mathbb{T}}$. In the case when $x(t)$ is eventually negative, the proof is similar. By Lemma 1 , we see that $x(t)$ satisfies either case (I) or case (II).

If case (I) holds, proceeding as in the proof of Theorem 9 , we get that (56) and (58) hold. We conclude that

$$
\begin{aligned}
& \frac{1}{H\left(\sigma(t), T_{0}\right)} \\
& \quad \times \int_{T_{0}}^{\sigma(t)}\left[H(\sigma(t), s) Q(s)-\frac{h_{-}^{\alpha+1}(t, s) r_{2}(s) \sigma^{\alpha^{2}}(s)}{(\alpha+1)^{\alpha+1} k^{\alpha^{2}} \tau^{\alpha^{2}}(s) \delta^{\alpha}(s)}\right] \Delta s \\
& \leq W\left(T_{0}\right) .
\end{aligned}
$$

From (88), we obtain

$$
\begin{gathered}
\Psi\left(T_{0}\right) \leq W\left(T_{0}\right), \quad T_{0} \in(T, \infty)_{\mathbb{T}} ; \\
\liminf _{t \rightarrow \infty} \frac{1}{H\left(\sigma(t), T_{0}\right)} \int_{T_{0}}^{\sigma(t)} H(\sigma(t), s) Q(s) \Delta s \geq \Psi\left(T_{0}\right),
\end{gathered}
$$

$$
\begin{gathered}
\liminf _{t \rightarrow \infty} \frac{1}{H\left(\sigma(t), T_{0}\right)} \int_{T_{0}}^{\sigma(t)} H(\sigma(t), s) Q(s) \Delta s \\
-\liminf _{t \rightarrow \infty} \frac{1}{H\left(\sigma(t), T_{0}\right)} \\
\quad \times \int_{T_{0}}^{\sigma(t)} \frac{h_{-}^{\alpha+1}(t, s) r_{2}(s) \sigma^{\alpha^{2}}(s)}{(\alpha+1)^{\alpha+1} k^{\alpha^{2}} \tau^{\alpha^{2}}(s) \delta^{\alpha}(s)} \Delta s
\end{gathered}
$$




$$
\begin{aligned}
\geq \liminf _{t \rightarrow \infty} & \frac{1}{H\left(\sigma(t), T_{0}\right)} \\
\times \int_{T_{0}}^{\sigma(t)}[H(\sigma(t), s) Q(s) & \\
& \left.\quad-\frac{h_{-}^{\alpha+1}(t, s) r_{2}(s) \sigma^{\alpha^{2}}(s)}{(\alpha+1)^{\alpha+1} k^{\alpha^{2}} \tau^{\alpha^{2}}(s) \delta^{\alpha}(s)}\right] \Delta s
\end{aligned}
$$$$
\geq \Psi\left(T_{0}\right)
$$

Using (87) and (92), we get

$\liminf _{t \rightarrow \infty} \frac{1}{H\left(\sigma(t), T_{0}\right)} \int_{T_{0}}^{\sigma(t)} \frac{h_{-}^{\alpha+1}(t, s) r_{2}(s) \sigma^{\alpha^{2}}(s)}{(\alpha+1)^{\alpha+1} k^{\alpha^{2}} \tau^{\alpha^{2}}(s) \delta^{\alpha}(s)} \Delta s<\infty$.

Thus, there exists a sequence $\left\{T_{n}\right\}_{n=1}^{\infty}: T_{n} \in\left[T_{0}, \infty\right)_{\mathbb{T}}$ with $\lim _{n \rightarrow \infty} T_{n}=\infty$ such that

$$
\lim _{n \rightarrow \infty} \frac{1}{H\left(\sigma\left(T_{n}\right), T_{0}\right)} \int_{T_{0}}^{\sigma\left(T_{n}\right)} \frac{h_{-}^{\alpha+1}\left(T_{n}, s\right) r_{2}(s) \sigma^{\alpha^{2}}(s)}{(\alpha+1)^{\alpha+1} k^{\alpha^{2}} \tau^{\alpha^{2}}(s) \delta^{\alpha}(s)} \Delta s<\infty .
$$

We define $A(t)$ and $B(t)$ also, as in the proof of Theorem 10 . From (56) and (91), we obtain

$$
\begin{aligned}
& \limsup _{t \rightarrow \infty}[B(t)-A(t)] \\
& \quad \leq W\left(T_{0}\right)-\liminf _{t \rightarrow \infty} \frac{1}{H\left(\sigma(t), T_{0}\right)} \int_{T_{0}}^{\sigma(t)} H(\sigma(t), s) Q(s) \Delta s \\
& \quad<\infty .
\end{aligned}
$$

For the above sequence $\left\{T_{n}\right\}_{n=1}^{\infty}$, we get

$$
\lim _{n \rightarrow \infty}\left[B\left(T_{n}\right)-A\left(T_{n}\right)\right] \leq \limsup _{t \rightarrow \infty}[B(t)-A(t)]<\infty .
$$

Similar to the proof of Theorem 10, we get (70). The rest proofs are the same as the Theorem 10, here omitted. This completes the proof.

Remark 12. From Theorems 9, 10, and 11, we can obtain different sufficient conditions for the oscillation of (1) with different choices of the functions $\delta$ and $H$.

Remark 13. The theorems in this paper are new even for the cases of $\mathbb{T}=\mathbb{R}$ and $\mathbb{T}=\mathbb{Z}$.

Example 14. Consider the third-order nonlinear delay dynamic equation

$$
\begin{gathered}
\left(t^{2 / 3}\left[\left(\frac{1}{t} x^{\Delta}(t)\right)^{\Delta}\right]^{5 / 3}\right)^{\Delta}+\frac{1}{t^{2}}\left(x\left(\frac{t}{2}\right)\right)^{5 / 3}\left(1+x^{2}\left(\frac{t}{2}\right)\right) \\
=0, \quad t \in \overline{2^{\mathrm{Z}}}, t \geq t_{0}:=2 .
\end{gathered}
$$

Here $r_{1}(t)=1 / t, r_{2}(t)=t^{2 / 3}, q(t)=1 / t^{2}, f(x)=x^{5 / 3}\left(1+x^{2}\right)$, $\tau(t)=t / 2<t$, and $\alpha=5 / 3$.

Conditions $\left(\mathrm{H}_{1}\right)-\left(\mathrm{H}_{3}\right)$ are clearly satisfied, and $\left(\mathrm{H}_{4}\right)$ holds with $L=1 . r_{2}^{\Delta}(t)=\left((2 t)^{2 / 3}-t^{2 / 3}\right) /(2 t-t)=\left(2^{2 / 3}-1\right) / t^{1 / 3}>0$, and

$$
\begin{gathered}
\int_{t_{0}}^{\infty} q(s) \Delta s=\int_{2}^{\infty} \frac{1}{s^{2}} \Delta s=\left[-\frac{2}{s}\right]_{2}^{\infty}=1<\infty, \\
\int_{t_{0}}^{\infty} \frac{1}{r_{1}(t)} \int_{t}^{\infty}\left[\frac{1}{r_{2}(s)} \int_{s}^{\infty} q(u) \Delta u\right]^{1 / \alpha} \Delta s \Delta t \\
=\int_{2}^{\infty} \frac{1}{t} \int_{t}^{\infty}\left[\frac{1}{s^{2 / 3}} \int_{s}^{\infty} \frac{1}{u^{2}} \Delta u\right]^{3 / 5} \Delta s \Delta t \\
=\int_{2}^{\infty} \frac{1}{t} \int_{t}^{\infty}\left[\frac{1}{s^{2 / 3}} \cdot \frac{2}{s}\right]^{3 / 5} \Delta s \Delta t \\
=2^{3 / 5} \int_{2}^{\infty} \frac{1}{t} \int_{t}^{\infty} \frac{1}{s} \Delta s \Delta t=\infty,
\end{gathered}
$$

so (26) holds.

Let $\delta(t)=t^{2}$, then $\delta^{\Delta}(t)=3 t \geq 0$. Since

$$
\begin{aligned}
Q(t) & =L q(t) \delta(\sigma(t))\left(\frac{k h_{2}\left(\tau(t), t_{0}\right)}{2 r_{1}(\tau(t)) \sigma(t)}\right)^{\alpha} \\
& =1 \cdot \frac{1}{t^{2}} \cdot(2 t)^{2} \cdot\left(\frac{k h_{2}\left(\tau(t), t_{0}\right)}{2 \cdot(2 / t) \cdot 2 t}\right)^{\alpha} \\
& =\frac{k^{5 / 3}}{8}\left(h_{2}\left(\tau(t), t_{0}\right)\right)^{5 / 3} \\
& \geq \frac{k^{5 / 3}}{8} h_{2}\left(\tau(t), t_{0}\right) \text { as } t \longrightarrow \infty, \\
\left.\frac{r_{2}(}{(}\right) & \delta(t) \\
(\alpha+1)^{\alpha+1} k^{\alpha^{2}} & {\left[\frac{\delta^{\Delta}(t)}{\delta(t)}\right]^{\alpha+1}\left[\frac{\sigma(t)}{\tau(t)}\right]^{\alpha^{2}} } \\
& =\frac{t^{2 / 3} \cdot t^{2}}{(8 / 3)^{8 / 3} \cdot k^{25 / 9}} \cdot\left(\frac{3 t}{t^{2}}\right)^{8 / 3}\left(\frac{2 t}{t / 2}\right)^{25 / 9} \\
& =\left(\frac{9}{8}\right)^{8 / 3} \cdot 4^{25 / 9} \cdot k^{-25 / 9},
\end{aligned}
$$

so that

$$
\begin{aligned}
& \limsup _{t \rightarrow \infty} \int_{t_{0}}^{t}\left\{Q(s)-\frac{r_{2}(s) \delta(s)}{(\alpha+1)^{\alpha+1} k^{\alpha^{2}}}\left[\frac{\delta^{\Delta}(s)}{\delta(s)}\right]^{\alpha+1}\left[\frac{\sigma(s)}{\tau(s)}\right]^{\alpha^{2}}\right\} \Delta s \\
& \geq \underset{t \rightarrow \infty}{\limsup } \int_{2}^{t}\left\{\frac{k^{5 / 3}}{8} h_{2}(\tau(s), 2)-\left(\frac{9}{8}\right)^{8 / 3}\right. \\
& \left.\cdot 4^{25 / 9} \cdot k^{-25 / 9}\right\} \Delta s
\end{aligned}
$$




$$
\begin{aligned}
& =\limsup _{t \rightarrow \infty}\left\{\frac{k^{5 / 3}}{8} \int_{2}^{t} h_{2}(\tau(s), 2) \Delta s-\left(\frac{9}{8}\right)^{8 / 3}\right. \\
& \left.\cdot 4^{25 / 9} \cdot k^{-25 / 9} \cdot \int_{2}^{t} \Delta s\right\} \\
& =\limsup _{t \rightarrow \infty}\left\{\frac{k^{5 / 3}}{8} \int_{2}^{t} 2 h_{2}(\tau(s), 2) \tau^{\Delta}(s) \Delta s-\left(\frac{9}{8}\right)^{8 / 3}\right. \\
& \left.\cdot 4^{25 / 9} \cdot k^{-25 / 9} \cdot \int_{2}^{t} \Delta s\right\} \\
& =\limsup _{t \rightarrow \infty}\left\{\frac{k^{5 / 3}}{4}\left[h_{3}(\tau(t), 2)-h_{3}(1,2)\right]-\left(\frac{9}{8}\right)^{8 / 3}\right. \\
& \left.\cdot 4^{25 / 9} \cdot k^{-25 / 9} \cdot(t-2)\right\} \\
& =\limsup _{t \rightarrow \infty}\left\{\frac{k^{5 / 3}}{4}\left[\frac{((t / 2)-2)((t / 2)-4)((t / 2)-8)}{21}+1\right]\right. \\
& \left.-\left(\frac{9}{8}\right)^{8 / 3} \cdot 4^{25 / 9} \cdot k^{-25 / 9} \cdot(t-2)\right\}=\infty .
\end{aligned}
$$

Then by Theorem 8 , every solution $x(t)$ of (97) is either oscillatory or converges to zero. But the other known results cannot be applied in (97).

\section{Conflict of Interests}

The authors declare that they have no conflict of interests regarding the publication of this paper.

\section{Acknowledgments}

The authors sincerely thank the reviewers for their valuable suggestions and useful comments that have led to the present improved version of the original paper. This research is supported by Shandong Provincial Natural Science Foundation (ZR2011AL001 and ZR2013AM003) and the Science Foundation of Binzhou University (BZXYQNLG201009).

\section{References}

[1] M. Bohner and S. H. Saker, "Oscillation of second order nonlinear dynamic equations on time scales," The Rocky Mountain Journal of Mathematics, vol. 34, no. 4, pp. 1239-1254, 2004.

[2] L. Erbe, "Oscillation criteria for second order linear equations on a time scale," The Canadian Applied Mathematics Quarterly, vol. 9, no. 4, pp. 345-375, 2001.

[3] L. Erbe, A. Peterson, and P. Rehák, "Comparison theorems for linear dynamic equations on time scales," Journal of Mathematical Analysis and Applications, vol. 275, no. 1, pp. 418-438, 2002.

[4] S. Sun, Z. Han, and C. Zhang, "Oscillation of second-order delay dynamic equations on time scales," Journal of Applied Mathematics and Computing, vol. 30, no. 1-2, pp. 459-468, 2009.
[5] Q. Zhang and L. Gao, "Oscillation criteria for second order halfliner delay dynamic equations with damping on time scales," Scientia Sinica A, vol. 40, no. 7, pp. 673-682, 2010 (Chinese).

[6] Q. Zhang, L. Gao, and S. Liu, "Oscillation criteria for second order half-liner delay dynamic equations with damping on time scales(II)," Scientia Sinica A, vol. 41, no. 10, pp. 885-896, 2011 (Chinese).

[7] S. R. Grace, R. P. Agarwal, B. Kaymakçalan, and W. Saejie, "Oscillation theorems for second order nonlinear dynamic equations," Journal of Applied Mathematics and Computing, vol. 32, no. 1, pp. 205-218, 2010.

[8] R. P. Agarwal, M. Bohner, and S. H. Saker, "Oscillation of second order delay dynamic equations," The Canadian Applied Mathematics Quarterly, vol. 13, no. 1, pp. 1-17, 2005.

[9] L. Erbe, A. Peterson, and S. H. Saker, "Oscillation criteria for second-order nonlinear delay dynamic equations," Journal of Mathematical Analysis and Applications, vol. 333, no. 1, pp. 505522, 2007.

[10] S. R. Grace, M. Bohner, and R. P. Agarwal, "On the oscillation of second-order half-linear dynamic equations," Journal of Difference Equations and Applications, vol. 15, no. 5, pp. 451-460, 2009.

[11] L. Erbe, A. Peterson, and S. H. Saker, "Asymptotic behavior of solutions of a third-order nonlinear dynamic equation on time scales," Journal of Computational and Applied Mathematics, vol. 181, no. 1, pp. 92-102, 2005.

[12] L. Erbe, A. Peterson, and S. H. Saker, "Hille and Nehari type criteria for third-order dynamic equations," Journal of Mathematical Analysis and Applications, vol. 329, no. 1, pp. 112131, 2007.

[13] Z. Han, T. Li, S. Sun, and M. Zhang, "Oscillation behavior of solutions of third-order nonlinear delay dynamic equations on time scales," Communications of the Korean Mathematical Society, vol. 26, no. 3, pp. 499-513, 2011.

[14] L. Gao, Q. Zhang, and S. Liu, "Oscillatory behavior of thirdorder nonlinear delay dynamic equations on time scales," Journal of Computational and Applied Mathematics, vol. 256, pp. 104-113, 2014.

[15] M. Bohner and A. Peterson, Dynamic Equations on Time Scales: An Introduction with Applications, Birkhäuser, Boston, Mass, USA, 2001.

[16] M. Bohner and A. Peterson, Advances in Dynamic Equations on Time Scales, Birkhäuser, Boston, Mass, USA, 2003.

[17] Y. Şahiner, "Oscillation of second-order delay differential equations on time scales," Nonlinear Analysis: Theory, Methods \& Applications, vol. 63, no. 5-7, pp. e1073-e1080, 2005.

[18] G. H. Hardy, J. E. Littlewood, and G. Pólya, Inequalities, Cambridge University Press, Cambridge, UK, 2nd edition, 1998.

[19] A. Tuna and S. Kutukcu, "Some integral inequalities on time scales," Applied Mathematics and Mechanics, vol. 29, no. 1, pp. 23-29, 2008. 


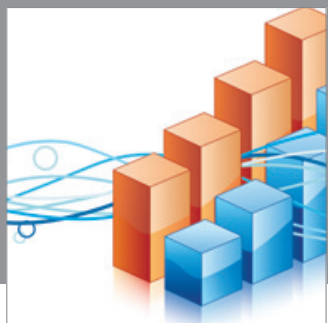

Advances in

Operations Research

mansans

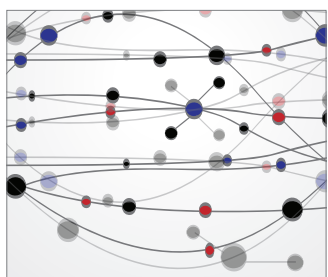

The Scientific World Journal
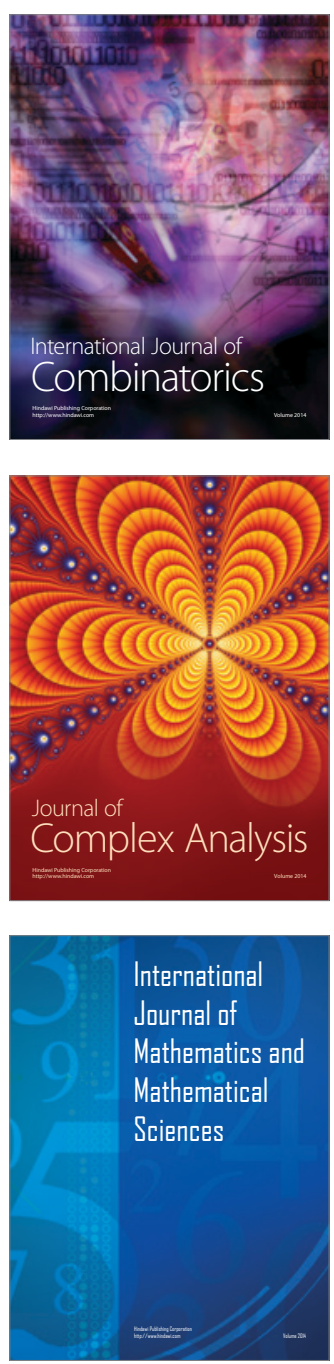
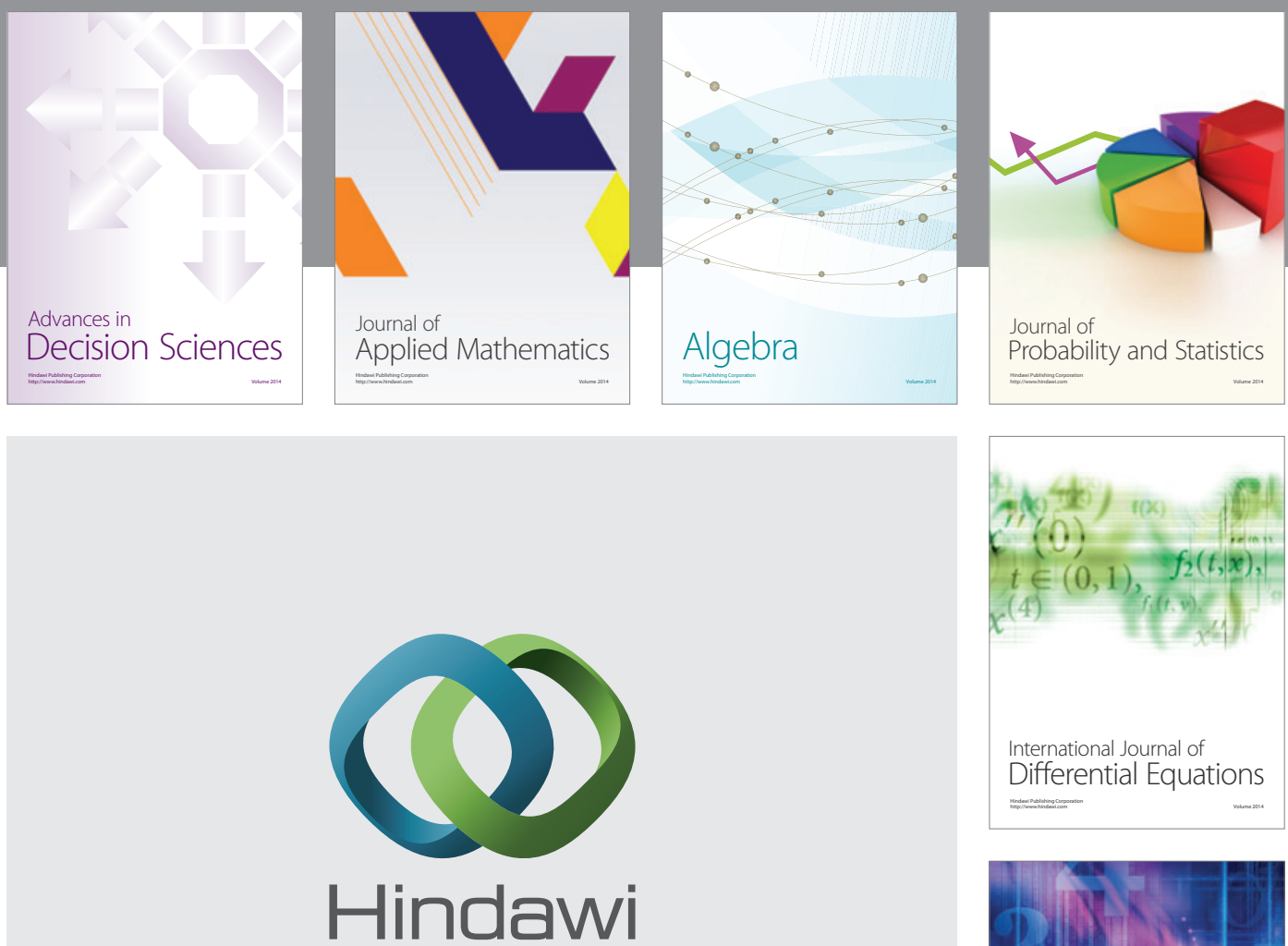

Submit your manuscripts at http://www.hindawi.com
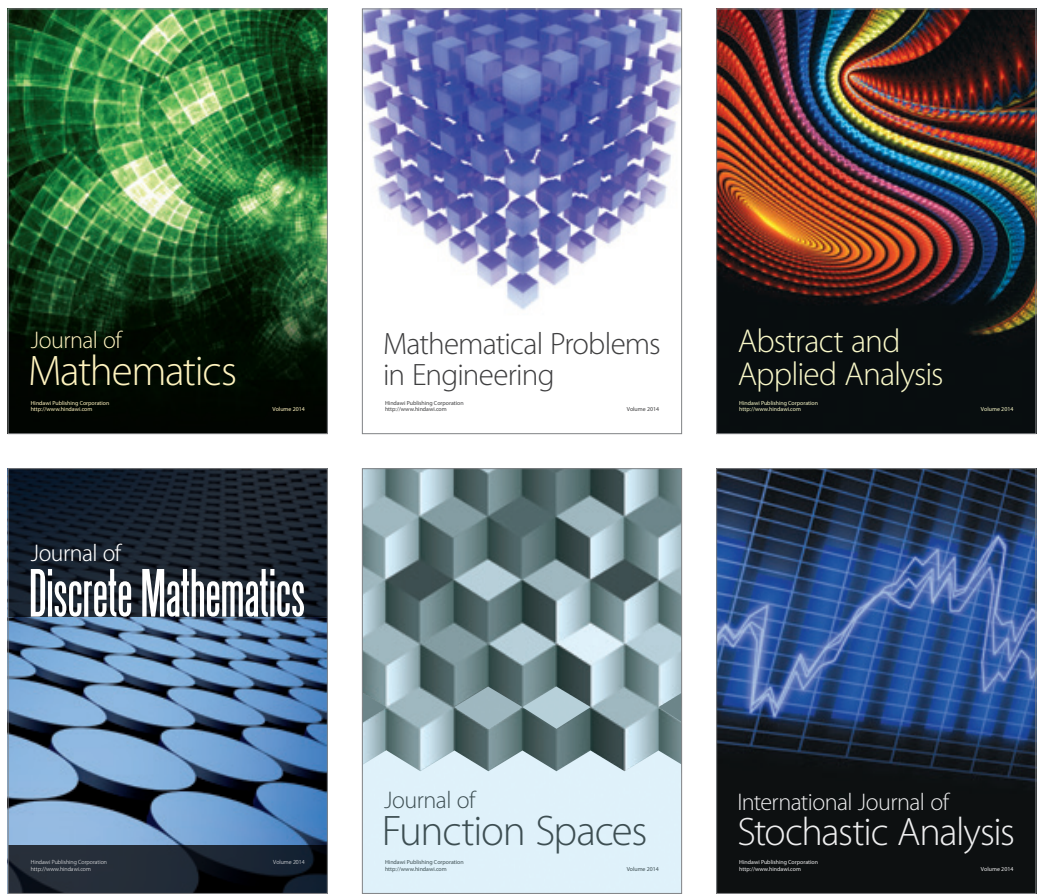

Journal of

Function Spaces

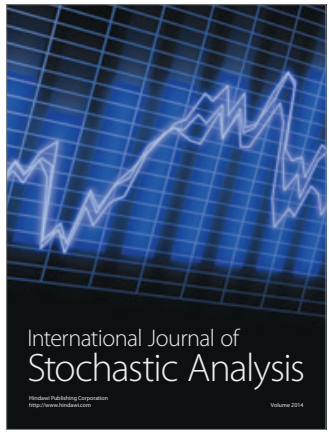

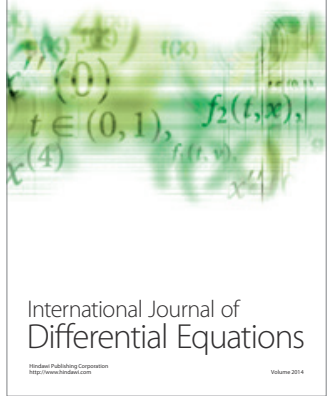
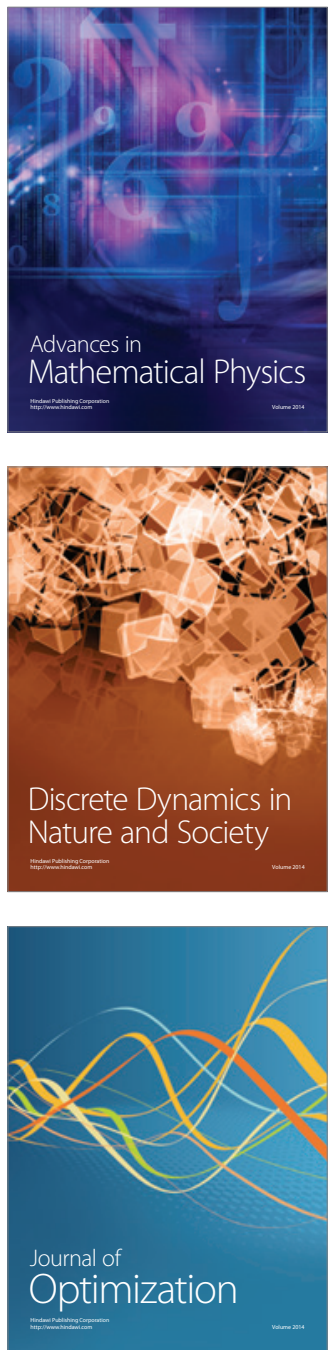